\title{
Pubic Osteolysis in an Elderly Masquerading as Malignancy: A Case Report and Review of Literature
}

\author{
Raju Vaishya ${ }^{1}$, Parv Mittal $^{1}$, Abhishek Vaish $^{1}$, Robin Khosa ${ }^{2}$
}

\section{Learning Point of the Article:}

Pelvic osteolysis and insufficiency fractures may occur after regional radiotherapy due to weakening of bones, especially by pre-existing Osteoporosis.

\section{Abstract}

Introduction: A rapidly progressive destructive lesion characterizes pubic osteolysis (PO) in the pubic bone due to an inadequate fracture healing response. It may be seen in pelvic insufficiency fractures (IF) secondary to radiation therapy (RT) of pelvic malignancies, occurring even in the absence of significant trauma. Such a radiological picture may distract the clinician towards a malignant etiology and may affect the management.

Case Report: A 79- year- old female, known case of carcinoma of the urinary bladder, underwent contrast-enhanced computed tomography (CT) (CECT) of the abdomen and pelvis as a routine follow- up and was found to have an osteolytic lesion in the right pubic bone, suggesting a malignant pathology. CT- guided biopsy did not reveal any malignant or infective etiology. The patient showed recovery with conservative management.

Conclusion: Osteolytic lesions of the pubic bone can often occur following radiation for pelvic malignancies. It occurs due to impaired fracture reparative response by a bone afflicted by radiation therapy RT. It can be managed effectively with conservative analgesics, bisphosphonates, calcium, and Vitamin D supplementation. The radiographic picture can imitate malignant or infective lesions and provoke invasive testing for confirmation. The clinicians need to be conscious of this clinical entity to initiate proper treatment and avoid unnecessary investigations.

Keywords: Pubic Osteolysis; Insufficiency Fracture; Radiation; Pelvis.

\section{Introduction}

A destructive lesion of the Pubis, following radiation therapy (RT) for pelvic malignancies such as uterus, cervix, anorectal, and bladder cancers may occur $[1,2,3,4,5,6,7,8,9]$. Pubic osteolysis (PO) is a subtype of an insufficiency fracture (IF) and may mimic a malignant lesion, as most of these cases may not have any history of trauma $[6,7,8,10]$. With a history of pelvic malignancy, the metastatic bone disease's suspicion remains a possibility and a concern. Therefore, it causes a diagnostic dilemma and compels the clinician to undertake invasive investigations like a biopsy to confirm the diagnosis. We present a case of a 79-year-old female with a history of Carcinoma of the Urinary Bladder, where a right pubic osteolytic lesion was found on a routine follow-up computed tomography (CT) scan, which was masquerading as a malignant tumor.

\section{Case Report}

A 79-years-old female came for routine follow-up investigations for her carcinoma of the urinary bladder (Fig. 1), which was diagnosed 15 months ago.

At present, she was asymptomatic and underwent contrastenhanced CT of the abdomen and pelvis, for a routine followup, which showed the tumor's complete resolution. This revealed osteolysis of the right pubic rami with a small fluid collection. Bilateral sacral IF were also noticed in the healing

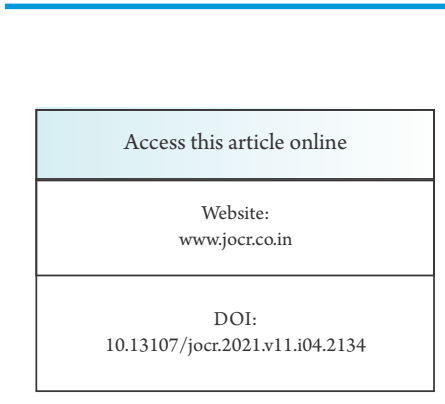

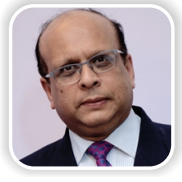

Dr. Raju Vaishya

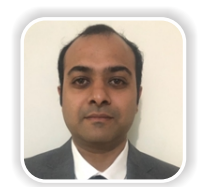

Dr. Parv Mittal

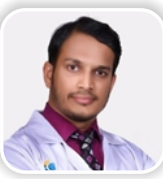

Dr. Abhishek Vaish

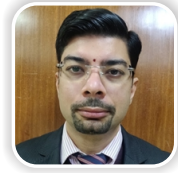

Dr. Robin Khosa
Department of Orthopaedics and Joint Replacement Surgery, Indraprastha Apollo Hospitals, New Delhi, India,

'Department of Radiation Oncology, Indraprastha Apollo Hospitals, New Delhi, India.

Address of Correspondence

Dr. Abhishek Vaish,

Department of Orthopaedics and Joint Replacement Surgery, Indraprastha Apollo Hospitals, New Delhi, India.

E-mail: drabhishekvaish@gmail.com

Journal of Orthopaedic Case Reports | pISSN 2250-0685 | eISSN 2321-3817 | Available on www.jocr.co.in | doi:10.13107/jocr.2021.v11.i04.2134 This is an Open Access article distributed under the terms of the Creative Commons Attribution Non-Commercial License (http://creativecommons.org/licenses/by-nc/3.0) which permits unrestricted non-commercial use, distribution, and reproduction in any medium, provided the original work is properly cited. 


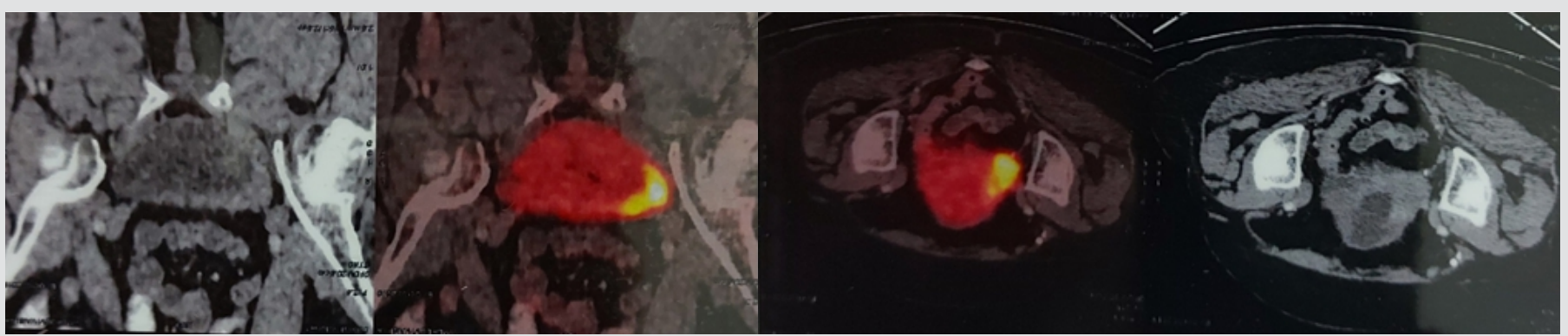

Figure 1: Positron emission tomography-computed tomography scans images confirming the malignant lesion in the urinary bladder, with an increased FDG uptake.

stage (Fig. 2a, b). A CT-guided biopsy was done of the public lesion, and $6 \mathrm{ml}$ of blood mixed fluid was aspirated. Cytolopathological examination did not reveal any malignant cells, nor there was any microbial growth. The Gene X-pert assay for Mycobacterium tuberculosis was negative. Her full blood counts and other laboratory investigations were within normal limits. A diagnosis of radiation-induced IF was thus made.

She had undergone combined chemotherapy and RT as a bladder preservation protocol and was given 6 weekly chemotherapy cycles (Cisplatin, $45 \mathrm{mg}$ ) and 64 Gy radiations in 32 cycles. Volumetric arc radiotherapy was given to the draining pelvic lymph nodes ( $46 \mathrm{~Gy} / 23$ fraction) and the whole bladder (54 Gy/27 fractions), followed by a booster dose to bladder tumor ( $10 \mathrm{~Gy} / 5$ fractions). To avoid the risk of pelvic IF (PIF), the sacral doses of radiation were limited to a mean dose of 29.6 Gy and each sacroiliac (SI) joint to a dose of $24.5 \mathrm{~Gy}$ (which is below the recommended dose of $<35 \mathrm{~Gy}$ ). Thr dose to pubic symphysis (drawn retrospectively) was higher a mean of 55.8 Gy and the maximum dose of $66.8 \mathrm{~Gy}$.

The last dose of RT was completed 13 months ago. Three months after completing the radiation, she developed persistent low back pain, which was moderately severe in intensity, and stopped walking without support. The Positron Emission Tomography-CT scan showed a diffuse increase in metabolic activity in the sacrum's bilateral ala, suggestive of an IF (Fig. 3). Magnetic resonance imaging (MRI) further confirmed a linear hyperintense line seen through the bilateral ala of sacrum representing IF (Fig. 4). Plain radiographs revealed generalized osteoporosis but did not show any fractures (Fig. 5). The DXA scan confirmed the diagnosis of osteoporosis, with a T-score of -2.6 .

As the patient refused any interventional treatment like C e m e n t a g m e n t a tion (Sacroplasty), she was treated

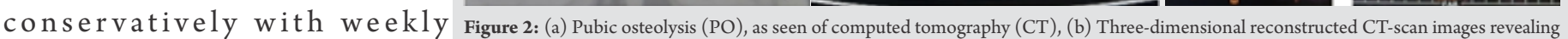

supplementation Vitamin D $(60,000$ right pubic osteolysis and healing bilateral sacral insufficiency fractures.

I.U) and sodium alendronate $(70 \mathrm{mg})$, along with calcium and analgesics. She responded to the conservative treatment slowly, and the pain vanished in about 6 months. After 2 months of the onset of low back pain, she felt some discomfort and stiffness in the groin, for which a plain radiograph was done, which did not reveal any pathological changes (Fig. 5). No specific treatment was given, and it settled along with low back pain. Since then, her mobility has been pain-free, and she did not require any walking support or painkillers.

\section{Discussion}

The PO is a subtype of IF with a destructive lesion of the pubic bone, following RT. It may be asymptomatic or present with pelvic pain and limp. Some cases of PO mimicking malignant lesions have been reported $[7,8,11,12,13,14,15]$. Radiationinduced PIF has a cumulative 5-year incidence ranging from $8.2 \%$ to $45.2 \%[1,2,3,4,9]$. These fractures have a higher incidence in postmenopausal females with osteoporosis, like in our case. The IF may present as subtle or apparent fractures (with or without callus formation) or destructive lesions. Most of the time, there is no history of antecedent trauma $[7,11,13$, 16].The weight-bearing bones (like Sacrum) tend to develop IF more commonly and earlier than the lesser weight-bearing bones (like Pubis), leading to earlier biomechanical failure in the sacrum. This fact is confirmed by the incidence of reported common fracture sites are SI joint (37.9\%), the body of the sacrum (33.9\%), and Pubis 13\% [17]. We believe that the initial sacral fracture in our case was followed by pubic bone
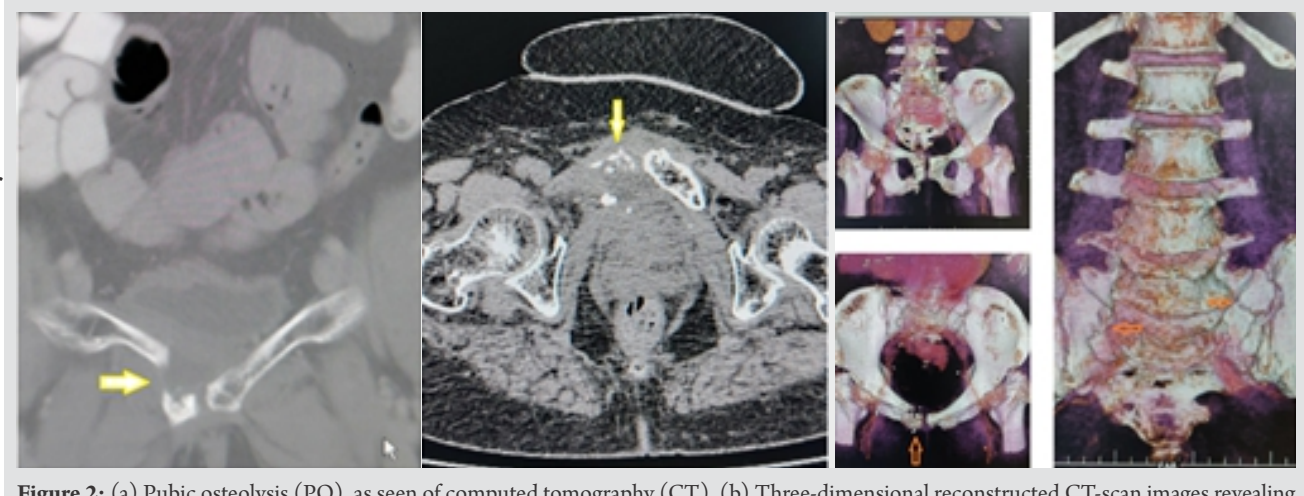


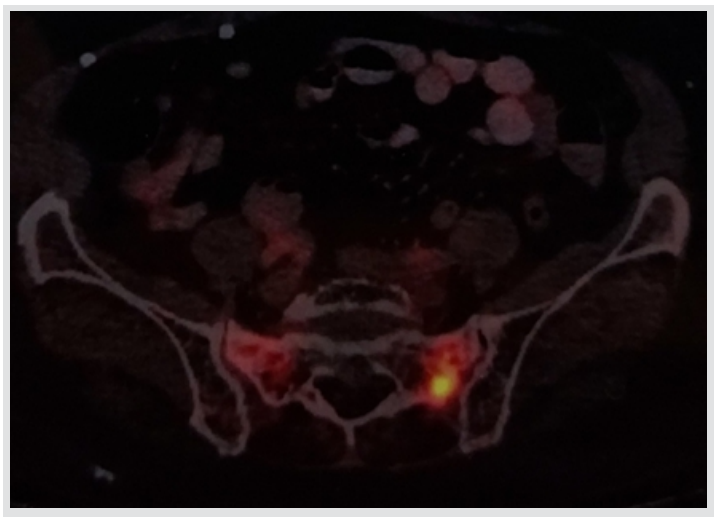

Figure 3: Positron emission tomography-computed tomography scan image showing increased FDG uptake in both ala of the sacrum ("Honda sign"), suggesting the presence of insufficiency fractures.

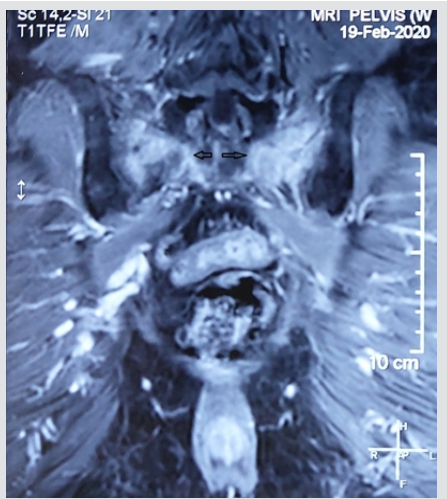

Figure 4: Bilateral insufficiency fractures of the ala of sacrum, as seen on magnetic resonance imaging scan.

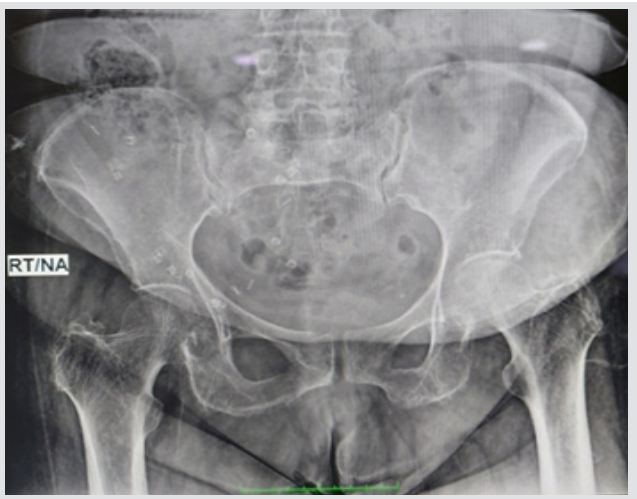

Figure 5: Antero-posterior radiograph of the pelvis, showing osteoporosis of both the hip bones, but no evidence of fractures in the pelvis. involvement after 9-10 months, perhaps due to excess loading of the pubic area while walking due to sacral pain.

The PO is a distinct clinical entity characterized by a rapidly progressive lesion seen mostly in postmenopausal females with the involvement of pubic bone and an absence of nuclear atypia on biopsy. The plain radiographs may not reveal the presence of IF, in the initial phase. MRI is highly sensitive in picking up the early lesions and also help in differentiating with the other mimicking lesions, such as (a) malignancy (e.g., primary chondrosarcoma, and metastatic bone disease), (b) infection/osteomyelitis (e.g., tuberculosis, and pyogenic), (c) non-infective inflammatory lesions (e.g., Rheumatoid Arthritis), and (d) non-specific lesions (e.g., osteitis pubis, steroid intake, and osteoporosis). Chondrosarcoma is a slowgrowing tumor, with a late presentation and male propensity, and rare involvement of the pubic bone [18]. It also shows the presence of nuclear polymorphism on biopsy. Osteomyelitis of pubic bone usually occurs as a postoperative complication of bladder surgeries or in cases of pelvic fractures with bladder rupture. It presents groin pain, pubic tenderness, draining sinus, fever, elevated Erythrocyte sedimentation rate, and total leukocyte counts. Radiographs may show the presence of a sequestrum. Osteitis pubis has a bimodal age distribution with professional athletes forming the younger cohort due to repeated microtrauma to the adductor longus tendon [19] and older patients with urinary tract infections, renal failure [20], or as a complication of bladder surgery. Its clinical presentation is with groin pain aggravated by hip abduction and adduction against resistance, limp and irregular margins, sclerosis, and pubic cleft widening on radiographs. Patients with bony metastasis from pelvic malignancies, for example, cervix, had a short survival time of around 7 months [21] with skeletal metastasis not more than $4 \%$ [22] and lower comparable rates of metastasis in the irradiated bone $[23,24,25]$.

Awareness about $\mathrm{PO}$ is crucial in reaching an early diagnosis and decreasing morbidity due to invasive investigations and surgical procedures, like hemipelvectomy [11, 15]. A high index of suspicion for $\mathrm{PO}$ is, therefore, required in patients undergoing $\mathrm{RT}$ for pelvic malignancies. The diagnosis of $\mathrm{PO}$ is often made as a coincidental finding on the radiographs, CT, or MRI. Mostly, symptomatic PO cases can be managed conservatively with rest, nonsteroidal anti-inflammatory drugs, calcium, Vitamin D supplementation, bisphosphonates, and graded physiotherapy.

\section{Conclusion}

The PO is an established clinical entity following RT for pelvic malignancies but is lesser known to the clinicians and poses a diagnostic challenge. Awareness of it is necessary to make an early diagnosis, decreasing morbidity, and avoiding unnecessary treatment. It is a benign condition that often remains asymptomatic and is detected as a coincidental finding on radiological studies. The symptomatic cases may have minimal discomfort, pain, and stiffness, which settle with conservative treatment, and surgery is not required in these cases.

\section{Clinical Message}

PO is a clinically described but unfamiliar condition arising due to impaired healing of IF occurring after radiotherapy. It often impersonates malignant lesions and may lead to nonrequired biopsy and surgical treatments. Mostly, it is diagnosed as a coincidental finding on radiological investigations and remains asymptomatic. 
1. Herman MP, Kopetz S, Bhosale PR, Eng C, Skibber JM, Rodriguez-Bigas MA, et al. Sacral insufficiency fractures after preoperative chemoradiation for rectal cancer: Incidence, risk factors, and clinical course. Int J Radiat Oncol Biol Phys 2009;74:818-23.

2. Abe H, Nakamura M, Takahashi S, Maruoka S, Ogawa Y, Sakamoto K. Radiation-induced insufficiency fractures of the pelvis: Evaluation with $99 \mathrm{mTc}$-methylene diphosphonate scintigraphy. AJR Am J Roentgenol 1992;158:599-602.

3. Ogino I, Okamoto N, Ono Y, Kitamura T, Nakayama H. Pelvic insufficiency fractures in postmenopausal woman with advanced cervical cancer treated by radiotherapy. Radiother Oncol 2003;68:61-7.

4. Ikushima H, Osaki K, Furutani S, Yamashita K, Kishida Y, Kudoh T, et al. Pelvic bone complications following radiation therapy of gynecologic malignancies: Clinical evaluation of radiation-induced pelvic insufficiency fractures. Gynecol Oncol 2006;103:1100-4.

5. Baxter NN, Habermann EB, Tepper JE, Durham SB, Virnig BA. Risk of pelvic fractures in older women following pelvic irradiation.JAMA 2005;294:2587-93.

6. Oh D, Huh SJ, Nam H, Park W, Han Y, Lim DH, et al. Pelvic insufficiency fracture after pelvic radiotherapy for cervical cancer: Analysis of risk factors. Int J Radiat Oncol Biol Phys 2008;70:1183-8.

7. Botton E, Saraux A, Malhaire JP, Mansourbakht T, Jousse S, Le Goff P, et al. Post-fracture osteolysis of the pubic bone simulating a malignancy: Report of a case. Joint Bone Spine 2004;71:230-3.

8. Ramón R, García S, Segur JM, Combalía A, Suso S. Pubic osteolysis simulating a malignant lesion: Case report. J Trauma 1994;36:597-98.

9. Vaishya R, Agarwal AK, Banka PK, Vijay V, Vaish A. Insufficiency fractures at unusual sites: A case series. J Orthop Case Rep 2017;7:76-9.

10. Park SH, Kim JC, Lee JE, Park IK. Pelvic insufficiency fracture after radiotherapy in patients with cervical cancer in the era of PET/CT. Radiat OncolJ 2011;29:269-76.

11. McCarthy B, Dorfman HD. Pubic osteolysis. A benign lesion of the pelvis closely mimicking a malignant neoplasm. Clin Orthop 1990;251:300-7.

12. Hall FM, Goldberg RP, Kadson EJ, Glick H. Post-traumatic osteolysis of the pubic bone simulating a malignant lesion.J Bone Joint Surg Am 1984;66:121-6.

13. Albertsen AM, Egund N, Jurik AG, Jacobsen E.
Posttraumatic osteolysis of the pubic bone simulating malignancy. Acta Radiol 1994;35:40-4.

14. An HS, Jackson WT, Hawthorne KB. Osteolysis of the pubic bone simulating malignancy. A case report and review of literature. Orthopedics 1988;11:951-4.

15. Goergen TG, Resnick D, Riley RR. Post-traumatic abnormalities of the pubic bone simulating malignancy. Radiology 1978;126:85-7.

16. Ramlov A, Pederson EM, Røhl L, Worm E, Fokdal L, Lindegaard JC, et al. Risk factors for pelvic insufficiency fractures in locally advanced cervical cancer following Intensity modulated radiation therapy. Int J Radiat Oncol Biol Phys 2017;97:1032-9.

17. Sapienza LG, Salcedo MP, Ning MS, Jhingran A, Klopp AH, Calsavara VF, et al. Pelvic insufficiency fractures after external beam radiation therapy for gynecologic cancers: A meta-analysis and meta-regression of 3929 patients. Int J Radiat Oncol Biol Phys 2020;106:475-84.

18. Dahlin DC, Unni KK. Bone Tumors: General Aspects and Data on 8,547 Cases. 4th ed. Springfield, Illnois: Charles C. Thomas; 1985.p. 30.

19. Harris NH, Murray RO. Lesions of the symphysis in athletes. Br Med J 1974;4:211-4.

20. Schabel SI, Burgener FA. Osteitis pubis in renal failure simulating chondrosarcoma. Br J Radiol 1975;48:1027-8.

21. Vermoken JB. The role of chemotherapy in squamous cell carcinoma of the uterine cervix: A review. Int J Gynaecol Cancer 1993;3:129-42.

22. Fagundes H, Perez CA, Grigsby PW, Lockett MA. Distant metastases after irradiation alone in carcinoma of the uterine cervix. Int J Radiat Oncol Biol Phys 1992;24:197204.

23. Hazra TA, Giri S. Prophylactic pelvic girdle irradiation in the treatment of prostatic carcinoma. Int J Radiat Oncol Biol Phys 1981;7:817-9.

24. Arafat QW, Davies AM. Parasymphyseal insufficiency fracture. Ann Rheum Dis 1994;53:421-4.

25. Matsumoto K, Hukuda S, Ishizawa M, Chano T, Okabe H, Maeno M. Pubic osteolysis mimicking a malignant lesion: Report a case with a fracture dislocation of the sacroiliac joint. Skeletal Radiol 1997;26:438-42. 


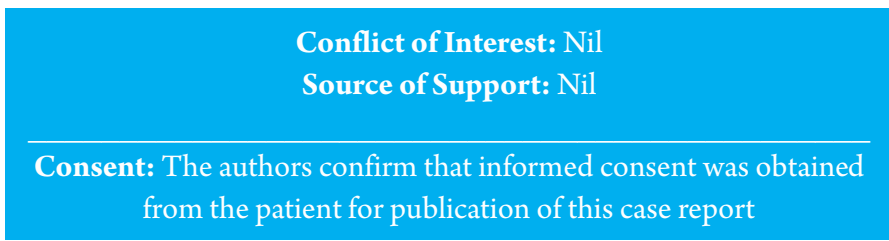

Conflict of Interest: Nil

Source of Support: Nil

from the patient for publication of this case report

\section{How to Cite this Article}

Vaishya R, Mittal P, Vaish A, Khosa R. Pubic Osteolysis in an Elderly Masquerading as Malignancy: A Case Report and Review of Literature. Journal of Orthopaedic Case Reports 2021 April;11(4): 18-22 